Historic, Archive Document

Do not assume content reflects current scientific knowledge, policies, or practices. 



\section{WHOLESALE PRICE LIST FALL 1919}

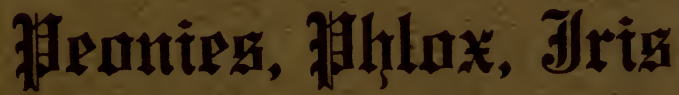

\section{anti ather pupular

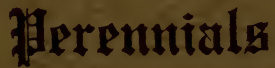

ORINOCO NURSERIES

A. B. CUNNINGHAM, PROP. COLUMBUS. - - INDIANA 



\section{CONDITIONS OF SALE}

\section{RATES}

Not less than 6 of a variety at dozen rate; 25 at 100 rate, or 250 at 1000 rate.

\section{PACKING}

All stock quoted herein will be packed free of charge, and with utmost care to insure arrival of stock in good condition.

\section{TERMS}

Cash, except to those of established credit, which, when known to us, the usual terms will be extended.

All Goods herein offered are subject to being unsold on receipt of order. My personal supervision is given to the growing and packing of all stock listed. 


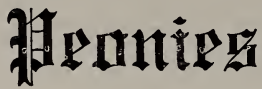

Prices given are for good strong divisions of 3 to 5 eyes. Our stock is carefully rozued during the blooming season and can be depended upon as being correctly labeled and unmixed.

Doz. $\quad 100$

Alexandre Dumas (Guerin)-Medium size, light pink; free bloomer. Early midseason $\ldots \ldots \ldots \ldots \ldots \ldots \ldots \ldots \ldots \ldots . \ldots 2.00 \$ 15.00$

Baroness Schroeder (Kelway) - Delicate flesh pink fading to milk white. Late midseason. Extra good for all purposes. Each $\$ 1.50 \ldots \ldots \ldots \ldots \ldots \ldots \ldots . \ldots . \ldots 15.00$

Charlotte Cushman (Hollis)_Bloom globular compact; uniform solferino red; large; late ......................

Couronne d'Or (Calot)_Large, white with yellow reflex. Late................ $3.00 \quad 25.00$

Dr. Bretonneau (Verdier) - Syn. Lady Bramwell. A beautiful silver-rose of fine form. Midseason...............

Dorchester (Richardson)-Large, compact, hydrangea pink. Late. Extra good....

Duke of Wellington (Calot)-Large, white with sulphur center. Late midseason. A good cut-flower variety.............

Festiva Maxima-Very large, pure white, flecked crimson; tall vigorous grower. Early. A great commercial sort....... $2.50 \quad 18.00$

Felix Crousse (Crousse)-A large, even, brilliant ruby red. Midseason.......... 
Doz. 100

Flo:al Treasure (Rosenfeld)-Large, full flowers of delicate pink; free bloomer and one of the best for commercial

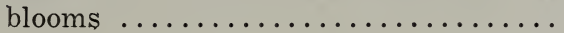

Golden Harvest (Rosenfield)-Peach blossom pink with creamy white center; midseason ................. 2.50

Jenny Lind (Barr)-Very large, hydrangea pink; plant tall, stems strong; free bloomer. Midseason. Fine commercial bloom $\ldots . . \ldots \ldots \ldots \ldots \ldots \ldots . . . \ldots$.

Karl Rosenfield (Rosenfield)-Very large, dark crimson, free bloomer; midseason. Plant tall, erect, growth strong and healthy. One of the best crimson varieties in the trade. Each $\$ 2.00 \ldots \ldots 20.00$

La Coquette (Guerin)-Guards and crown light pink, collar rose white, center flecked carmine; fragrant. Midseason. .

Livingstone (Crousse)-Buds and flowers are large and beautiful; pale lilac rose; free bloomer. Late..............

Louis Van Houtte (Calot)-Rich, dark crimson; free bloomer. Late midseason $2.00 \quad 15.00$

Madam de Verneville (Crousse)-Large, full, pure white with carmine tipped central petals. Early.............. $2.50 \quad 18.00$

Meissonier (Crousse)-Uniform dark brilliant red; free bloomer. Midseason. Good commercial bloom.............

Noemie Demay (Calot)_Large, blush pink fiowers borne on strong stems. Midseason to late. 
Doz. $\quad 100$

Perfection (Richardson)-Medium to large full double, very pale pink. Late bloomer. Very fine.............. $3.50 \quad 25.00$

Princess Beatrice (Kelway)-Guards and crown light pink, collar creamy white, center flecked crimson. Vigorous grower, free bloomer, fragrant. Midseason....

Peonies, single, separate colors in pink

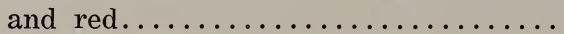

Peonies-Mixture of named varieties, red, white and pink, double; $\$ 70.00$ per 1000

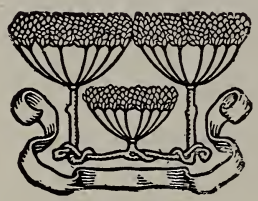




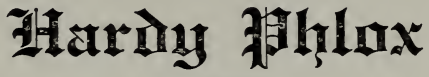

A recent visitor at our nursery, after placing an order for several thousand Phlox, said: "I could have bought at lower prices but I wanted stock true and unmixed."

Following varieties strong one-year plants: $\$ 6.00$ per 100; $\$ 50.00$ per 1000 .

Beranger-White suffused rosy pink.

Blanch Nana-A beautiful pure white of dwarf habit. Bridesmaid-White, crimson eye. Tall.

Duguesclin-Rosy lilac, rayed with lighter halo.

Eclaireur-Tall, bright rosy magenta with large, lighter halo; large florets.

Independence-Tall, early, pure white.

Jules Cambon-Carmine amaranth, large white center; dwarf.

La Vogue-Pure mauve rose with analine red eye.

Richard Wallace-Pure white with violet rose eye; large trusses.

R. P. Struthers-Clear cherry red, suffused with salmon shades; deep red eye.

The following varieties at $\$ 7.00$ per $100 ; \$ 60.00$ per 1000.

Rynstrom-Soft salmon rose. One of the best of pinks.

Von Hochberg-Large, rich velvety crimson. Distinct.

Von Lassburg-Flowers and trusses largest of any white. 


\section{Frizis (Fleur de Lis)}

Strong tubers, 50c per doz.; $\$ 3.00$ per $100 ; \$ 25.00$ per 1000.

Celeste-Pale azure blue.

Her Majesty-Standards bright lavender rose; falls crimson over white, giving a slightly striped effect. 75 c per doz.

Honorabilis-Stands golden yellow; falls mahozany brown.

John de Witt-Stands bluish violet; falls deep violet purple.

L'Avenir-Falls and standards ageratum blue, brown veins.

Madam Chereau-Pure white, prettily frilled and penciled with light clear blue. One of the best of the taller sorts.

Pallida Speciosa-Stands rich lavender; falls shaded light purple.

Flicata-Stands bright violet purple; falls deep violet purple.

Flumeri-Standards and falls deep coppery red. Early and free flowering.

Queen of May-A lovely rose lilac, almost pink. Beautiful.

Spectabilis-Stands light lavender purple; falls deep violet purple. Very early.

Virgile-Stands light blue; falls violet blue, veined.

Siberian Iris, Blue-Flowers a beautiful shade of deep blue.

Siberian Iris, Snow Queen-Pure white. 


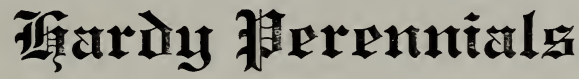

Doz. $\quad 100$

Achillea Millefolium Roseum.......... .75 5.00

-The Pearl-True double flowering.... $\quad .75 \quad 5.00$

Aster Novae Angliae-Purple and rose... $\quad .85 \quad 6.00$

Aquilegia Canadensis, Chrysantha and

Coerulea ...................... .85 6.00

Callicaı pa Purpurea, 2 yr........... 1.50

Chrysanthemum, large, flowered hardy sorts in red, pink, white and yellow.... $1.00 \quad 7.00$

Coreopsis Grandiflora............... . .75 5.00

Delphinium Belladonna, 1 year......... $.100 \quad 7.00$

-Chinese Blue................ .85 6.00

—Gold Medal Hybrids............. $\quad .85$

Dianthus Plumarius (Hardy Pinks)..... $\quad .85 \quad 6.00$

Digitalis Grandiflora (Foxglove)....... $\quad .85 \quad 6.00$

Euphorbia Corollata (Flowering Spurge) $\quad .75 \quad 5.00$

Funkia Coerulea and Variegata........ $\quad .75$

Gaillardia Grandiflora.............. $\quad .75 \quad 5.00$

Gypsophila Paniculata............ $\quad .85$

Grasses, Ornamental-

-Erianthus Ravennae............. 1.00

—Eulalia Variegata............. 1.00

-Pennisetum Japonica............. 1.00

Hemerocallis Flava and Thunbergi...... $\quad .75 \quad 5.00$

Iberis Sempervirens (Hardy Candytuft) . $\quad 1.00 \quad 7.00$

Hollyhock, double pink, red and white... $\quad .75 \quad 5.00$

Lavendula Vera (Sweet Lavender)...... $\quad .85$

Penstemon Barbatus Torreyi........... . . . $55 \quad 5.00$

Sedum Spectabilis................. .75 5.00

Shasta Daisy, Alaska..................... $85 \quad 5.00$

Sweet William, pink, red and white...... $\quad .75 \quad 5.00$

Veronica Spicata ................ $\quad .75 \quad 5.00$ 

$1=-$

$\sqrt{2}$

$3^{2}=$

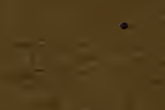
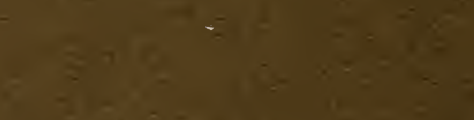